Original Article

\title{
Correlation among sarcopenia, malnutrition and activities of daily living in patients with vertebral compression fractures: a comparison based on admission and discharge parameters evaluating these conditions
}

\author{
Kohei TaKahashi, RPT, MS ${ }^{1)^{*}}$, Akira Kubo, RPT, PhD ${ }^{2)}$, KaYoko Ishimura, RPT ${ }^{1)}$, \\ TAKAhiro FukUi, RPT ${ }^{1)}$, Tetsuro TAMUra, MD $^{1)}$ \\ 1) Department of Rehabilitation, Tamura Surgical Hospital: 1-9-13 Tode, Saiwai-ku, Kawasaki-shi, \\ Kanagawa 212-0005, Japan \\ 2) Department of Physical Therapy, Faculty of Health Science, International University of Health and \\ Welfare, Japan
}

\begin{abstract}
Purpose] Sarcopenia may be associated with malnutrition in patients with vertebral compression fractures which may affect a patient's functional prognosis. This study investigated the association between sarcopenia, malnutrition, and activities of daily living at the time of hospital discharge in patients with vertebral compression fractures. [Participants and Methods] The study included 36 patients who were hospitalized with vertebral compression fractures. Sarcopenia was assessed by measuring grip strength and calf circumference. The nutritional status was assessed at the time of hospital admission and at discharge using the Mini Nutritional Assessment Short Form screening tool. Activities of daily living were assessed using the Barthel Index. [Results] The prevalence of sarcopenia at the time of admission was 47.2\%. The Barthel Index and Mini Nutritional Assessment Short Form scores in patients with sarcopenia at the time of admission were significantly lower at discharge than to those in patients without sarcopenia. Overall, at discharge, weight and calf circumference decreased significantly with a consequent increase in the prevalence of sarcopenia (55.6\%). Multivariate analysis showed that the Mini Nutritional Assessment Short Form score, calf circumference loss, and age affected the Barthel Index at discharge. [Conclusion] Patients with vertebral compression fractures often show sarcopenia and malnutrition, which are conditions that may be exacerbated during hospitalization. These conditions can subsequently affect a patient's activities of daily living; thus, nutritional rehabilitation is important in patients with vertebral compression fractures, as demonstrated in this study.

Key words: Vertebral compression fracture, Sarcopenia, Malnutrition
\end{abstract}

(This article was submitted Jul. 9, 2018, and was accepted Sep. 12, 2018)

\section{INTRODUCTION}

In Japan, the number of patients with vertebral compression fracture (VCF) has tended to increase because of the superaging of society. There are approximately 2 million cases of VCF per year ${ }^{1)}$, and this fracture causes pain and dysfunction, reduces QOL, and increases the risk of death ${ }^{2,3)}$. Therefore, future countermeasures against VCF are important.

Patients with VCF also often exhibit sarcopenia, which is defined as a syndrome characterized by a progressive and

*Corresponding author. Kohei Takahashi (E-mail: kohei_t504@yahoo.co.jp)

(C2018 The Society of Physical Therapy Science. Published by IPEC Inc.

(c) (1) $\odot$ This is an open-access article distributed under the terms of the Creative Commons Attribution Non-Commercial No DerivaCC BY NC ND tives (by-nc-nd) License. (CC-BY-NC-ND 4.0: https://creativecommons.org/licenses/by-nc-nd/4.0/) 
systemic decrease of skeletal muscle mass and strength, along with physical dysfunction and a risk of death ${ }^{4}$. Sarcopenia progresses due to aging, decreased activities, malnutrition, and disease ${ }^{4}$. Patients with VCF have significantly decreased muscle mass ${ }^{5,6)}$, and the prevalence of sarcopenia in these patients is $22.7-43.7 \%{ }^{7-9)}$, which is higher than that of other orthopedic disorders ${ }^{9}$. Some patients with VCF may also develop anorexia and subsequent malnutrition due to the pain and deformation of the spine after onset of the disease ${ }^{10)}$, and malnutrition affects the outcome of rehabilitation ${ }^{11,12)}$. In elderly women with acute VCF, nutritional intake is also associated with $\mathrm{ADL}^{13}$ ), andmalnutrition is a significant predictor for mortality after $\mathrm{VCF}^{14}$ ) . These findings suggest that sarcopenia may be associated with malnutrition in patients with $\mathrm{VCF}$, which may affectfunctional prognosis. However, there are few data available for this relationship.

Therefore, the purpose of this study is to investigate associations among sarcopenia, malnutrition, and ADL in patients with VCF.

\section{PARTICIPANTS AND METHODS}

A retrospective cohort study was performed in patients aged $\geq 65$ years who were admitted to Tamura Surgical Hospital with VCF from February 2016 to August 2017. During this period, 52 such patients were hospitalized. Patients were excluded if they could not walk before suffering the fracture ( 5 cases), had missing data (3 cases), had difficulty in communication ( 4 cases), had a neurological disorder in the lower extremities (2 cases), or were transferred to another hospital due to development of a malignant tumor or a complication (2 cases). Therefore, 36 patients (male: 11, female: 25, age: $83.6 \pm 5.5$ years, mean $\pm \mathrm{SD}$ ) with VCF were included in the final analysis.

The participants were divided into groups with and without sarcopenia at admission. It was difficult to obtain walking speeds for each participant at hospital admission because some also suffered from lumbar pain. Therefore, evaluation of sarcopenia was performed based on grip strength and calf circumference (CC) at admission (Fig. 1). Grip strengths of the right and left hands were measured twice each using a Smedley-type hand dynamometer, and the maximum value was recorded. The standard values of the Asian Working Group for Sarcopenia are $26 \mathrm{~kg}$ for males and $18 \mathrm{~kg}$ for females ${ }^{15}$, with a grip strength lower than these values defined as low. The CC was measured using Inser-Tape (Abbott Japan Co., Ltd., Tokyo, Japan) in the maximum bulging area in a supine position with the knee joint bent by $90^{\circ}$. The mean of measurementsfor the right and left leg was calculated. Amean $\mathrm{CC} \leq 30 \mathrm{~cm}$ for males and $\leq 29 \mathrm{~cm}$ for females was defined as low, as suggested by Maeda et $\mathrm{al}^{16)}$.

Nutritional status at admission and at discharge was evaluated using the Mini Nutritional Assessment Short Form (MNA$\mathrm{SF})^{17}$ ), with scores (range 1-14) of $\geq 12,8-11$, and $<8$ points defined as a favorable nutritional status, risk of malnutrition, and malnutrition, respectively. The daily energy intake rate was calculated by a dietician for 7 days after admission by dividing energy intake by energy consumption. Energy obtained from intravenous nutrition and/or nutritional supplements was added to the energy intake. Energy consumption was obtained using the following formula: basal metabolic expenditure calculated with the Harris-Benedict Equation $\times 1.2$ (activity index).

Body weight at admission was obtained from the patient or based on information from their family members. Weight at discharge was measured in a standing position on a scale. Height was measured when the patient became able to take a standing position. Changes in weight and CC during the period from admission to discharge were evaluated as follows:

Weight loss $(\%)=($ weight at admission - weight at discharge $) /$ weight at admission $\times 100$.

$\mathrm{CC}$ loss $(\%)=(\mathrm{CC}$ at admission $-\mathrm{CC}$ at discharge $) / \mathrm{CC}$ at admission $\times 100$.

$\mathrm{ADL}$ at admission and discharge was measured using the Barthel Index (BI). Walking ability before admission, at the start of gait training, and at discharge was evaluated using the Functional Ambulation Category (FAC) ${ }^{18)}$. Values were classified into 6 levels based on quality of walking, ranging from the assistance-needed level to the highest level. Pain at admission and

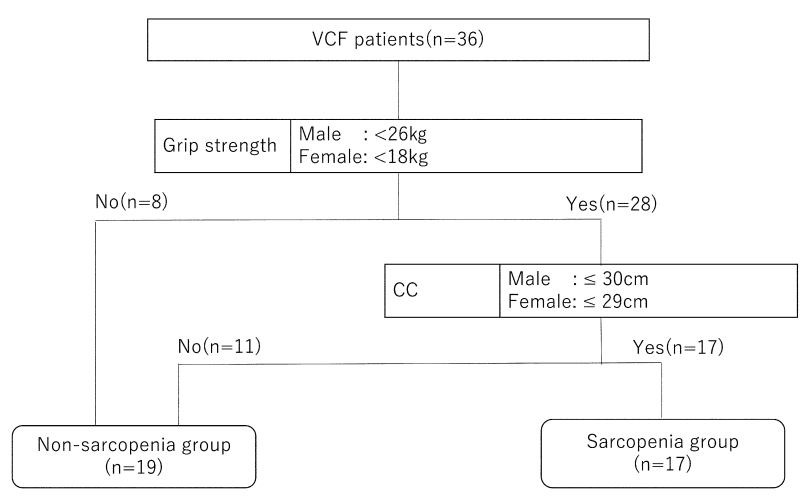

Fig. 1. Diagnosis of sarcopenia.

VCF: Vertebral compression Fracture; CC: Calf circumference. 
at discharge was evaluated using the Numerical Rating Scale (NRS) as a score from 0 to 10 , indicating no pain to the severest pain. The number of preparatory days beforegait training and the hospitalization days were also recorded.

Histories of the participants are based on hospital medical records. Total protein (TP) and C-reactive protein (CRP) were measured in blood tests at admission. Fractures were classified into each crush injury level based on a plain X-ray examination at admission using the semiquantitative (SQ) method ${ }^{19)}$, and the number of vertebral fractures was evaluated.

Physical therapy was given to all participants. Because low back pain was strong at admission, physical therapy began with relaxation, range of motion exercise, and resistance training at the bedside. If pain was relieved, treatment was advanced to sitting, standing, ADL, and walking training. The patient wore a corset as needed. Progression of activities was dependent on patient tolerance and stability.

Comparison of the sarcopenia and non-sarcopenia groups was made by unpaired t-test or Mann-Whitney U test after confirming the normality of variables in a $\chi^{2}$ test for nominal scales, a Mann-Whitney U test for ordinal scales, and a ShapiroWilk test for continuous variables. Weight and CC at admission and at discharge were evaluated by paired t-test, and the number of participants with sarcopenia and/or malnutrition was compared by signed rank test. Spearman rank correlation analysis was used to examine relationships between BI and other items. To identify factors that affected ADL at discharge, stepwise regression analysis was conducted utilizing BI at discharge as the dependent variable. Variables with a significant association with BI in univariate analysis were selected as independent variables. All statistical analyses were performed using SPSS Statistics 23 (IBM), and the significance level was 5\%.

The participants were provided with an explanation of the study objectives and measurements. The study was conducted after obtaining approval from the ethical committee of the International University of Health and Welfare (Approval Number: 16-Ig-41).

\section{RESULTS}

A comparison of the sarcopenia and non-sarcopenia groups is shown in Table 1. Of the 36 participants, 17 (47.2\%) had sarcopenia and $22(61.1 \%)$ had malnutrition. Compared to the non-sarcopenia group, the sarcopenia group had a significantly lower BI and walking ability at discharge, and had a significantly lower BMI and MNA-SF at both admission and discharge. Changes in sarcopenia and malnutrition from admission to discharge are shown in Table 2. At discharge, there were significant decreases in weight and CC in all participants, and 20 (55.6\%) had sarcopenia and 24 (66.7\%) had malnutrition, showing a tendency for these conditions to increase. Spearman rank correlations for BI at discharge are shown in Table 3. BI at discharge had significant correlations with age, BMI, presence of sarcopenia, MNA-SF scores at admission and at discharge, FAC at the start of gait training and at discharge, weight loss, and CC loss $(\mathrm{p}<0.05)$. The results of multiple linear regression analysis for BI at discharge are shown in Table 4. MNA-SF score at admission, CC loss, and age emerged as independent factors affecting BI at discharge.

Table 1. Sarcopenia group and non-sarcopenia group outcomes

\begin{tabular}{lccc}
\hline & Overall & Non-sarcopenia & Sarcopenia \\
\cline { 2 - 4 } & $(\mathrm{n}=36)$ & $(\mathrm{n}=19)$ & $(\mathrm{n}=17)$ \\
\hline Basic information & & & $82.5 \pm 6.2$ \\
Age (years) & $83.6 \pm 5.5$ & $84.5 \pm 4.8$ & 6 \\
Gender & & & 11 \\
Males (n) & 11 & 5 & 2 \\
Females (n) & 25 & 14 & 12 \\
Medical histry & & & 3 \\
Stroke (n) & 5 & 3 & 2 \\
Fracture (including other than VCF) (n) & 20 & 8 & 3 \\
Heart disease (n) & 8 & 5 & 1 \\
Respiratory disease (n) & 3 & 1 & $3[2-3]$ \\
Dementia (n) & 5 & 2 & $2.5 \pm 0.9$ \\
Diabetes (n) & 3 & 2 & $4[4-5]$ \\
Grade of SQ method & $3[1-3]$ & $3[1-3]$ & $1.9 \pm 1.0$ \\
Number of VCF (piece) & $2.2 \pm 0.6$ & $5[4-5]$ & \\
FAC before hospitalization & $4[4-5]$ & & \\
\hline
\end{tabular}


Table 1. Continued.

\begin{tabular}{|c|c|c|c|}
\hline & Overall & Non-sarcopenia & Sarcopenia \\
\hline & $(n=36)$ & $(n=19)$ & $(\mathrm{n}=17)$ \\
\hline \multicolumn{4}{|l|}{ At admission } \\
\hline BI (score) & $21.5 \pm 10.5$ & $21.8 \pm 12.8$ & $21.2 \pm 7.6$ \\
\hline Hight (cm) & $152.2 \pm 9.2$ & $152.8 \pm 7.3$ & $151.5 \pm 11.2$ \\
\hline Weight (kg) & $47.4 \pm 9.6$ & $54.1 \pm 6.9$ & $39.8 \pm 5.7^{* *}$ \\
\hline BMI $\left(\mathrm{kg} / \mathrm{m}^{2}\right)$ & $20.5 \pm 3.8$ & $23.2 \pm 2.6$ & $17.4 \pm 2.4^{* *}$ \\
\hline Grip strength (kg) & $15.6 \pm 7.6$ & $18.7 \pm 8.5$ & $12.2 \pm 4.7^{* *}$ \\
\hline $\mathrm{CC}(\mathrm{cm})$ & $29.0 \pm 3.0$ & $31.2 \pm 1.7$ & $26.5 \pm 2.0^{* *}$ \\
\hline MNA-SF (score) & $6.3 \pm 2.2$ & $7.7 \pm 1.5$ & $4.7 \pm 1.8^{* *}$ \\
\hline Malnutrition (n) & 22 & 6 & $16^{* *}$ \\
\hline At risk of malnutrition (n) & 14 & 13 & $1^{* *}$ \\
\hline Well nourished & 0 & 0 & 0 \\
\hline $\mathrm{TP}(\mathrm{g} / \mathrm{dl})$ & $6.7 \pm 0.7$ & $6.7 \pm 0.6$ & $6.8 \pm 0.8$ \\
\hline CRP (mg/dl) & $1.8 \pm 1.8$ & $1.6 \pm 1.6$ & $2.0 \pm 2.1$ \\
\hline Energy intake rates & $1.0 \pm 0.3$ & $1.0 \pm 0.3$ & $1.0 \pm 0.2$ \\
\hline Pain & $8.0 \pm 1.5$ & $8.2 \pm 1.6$ & $7.8 \pm 1.5$ \\
\hline \multicolumn{4}{|l|}{ Process } \\
\hline The days required to begin gait training (days) & $12.0 \pm 6.4$ & $12.0 \pm 7.1$ & $11.9 \pm 5.7$ \\
\hline FAC at the start of gait training & $3[1-4]$ & $3[1-4]$ & $2[1-3]^{* *}$ \\
\hline \multicolumn{4}{|l|}{ At discharge } \\
\hline BI (score) & $76.9 \pm 18.1$ & $84.5 \pm 13.8$ & $68.5 \pm 19.0^{* *}$ \\
\hline FAC & $4[1-5]$ & $4[3-5]$ & $4[1-4]^{*}$ \\
\hline Weight (kg) & $45.0 \pm 8.3$ & $50.1 \pm 5.6$ & $38.3 \pm 5.3^{* *}$ \\
\hline $\mathrm{BMI}\left(\mathrm{kg} / \mathrm{m}^{2}\right)$ & $19.4 \pm 3.5$ & $21.9 \pm 2.5$ & $16.7 \pm 2.3^{* *}$ \\
\hline Grip strength $(\mathrm{kg})$ & $15.7 \pm 7.8$ & $18.9 \pm 8.9$ & $12.1 \pm 4.1^{* *}$ \\
\hline $\mathrm{CC}(\mathrm{cm})$ & $28.3 \pm 2.9$ & $30.4 \pm 1.9$ & $25.9 \pm 1.7^{* *}$ \\
\hline MNA-SF (score) & $6.7 \pm 2.4$ & $7.8 \pm 2.2$ & $5.3 \pm 1.8^{* *}$ \\
\hline Malnutrition (n) & 24 & 9 & $15^{* *}$ \\
\hline At risk of malnutrition (n) & 12 & 10 & $2 * *$ \\
\hline Well nourished & 0 & 0 & 0 \\
\hline Pain & $3.0 \pm 2.6$ & $2.8 \pm 1.9$ & $3.2 \pm 2.6$ \\
\hline Weight loss (\%) & $4.7 \pm 5.2$ & $5.5 \pm 6.0$ & $3.8 \pm 4.3$ \\
\hline CC loss $(\%)$ & $2.3 \pm 3.3$ & $2.6 \pm 3.4$ & $1.9 \pm 3.2$ \\
\hline The number of hospitalization days (days) & $33.8 \pm 14.0$ & $32.5 \pm 12.0$ & $35.3 \pm 16.3$ \\
\hline
\end{tabular}

Mean $\pm \mathrm{SD}$, median [range].

VCF: Vertebral Compression Fracture; SQ: Semiquantitative Method; FAC: Functional Ambulation Categories; BMI: Body Mass Index; CC: Calf circumference; BI: Barthel Index; MNA-SF: Mini Nutritional Assessment-Short Form; TP: Total Protein; CRP: C-reactive protein.

Comparison of non-sarcopenia group and sarcopenia group. Continuous variables: unpaired t-test; Mann-Whitney U-test. Categorical variables: $\chi^{2}$ test. ${ }^{*} \mathrm{p}<0.05,{ }^{* *} \mathrm{p}<0.01$.

\section{DISCUSSION}

This study of the relationships among sarcopenia, nutritional status and ADL in patients with VCF resulted in three findings. First, patients with sarcopenia had poor nutritional status and low ADL at discharge. Second, weight loss and muscle atrophy were found in many of the patients at discharge. Third, malnutrition, CC loss, and age are associated with ADL at discharge.

Sarcopenia and malnutrition were present at rates of $47.2 \%$ and $61.1 \%$ in the patients in this study. A prevalence of sarcopenia in patients with VCF of $22.7-43.7 \%$ has been reported in previous studies ${ }^{7-9}$ ). The higher rate in the current study might be due to the higher age of the participants and to the inclusion of more participants with multiple VCF. There are few previous data on the rate of malnutrition in patients with VCF, but the results of this study suggest that such patients are highly likely to develop malnutrition as a complication. In addition, nutritional status was poorer in patients with sarcopenia. 
Table 2. Changes in sarcopenia and malnutrition at admission and discharge

\begin{tabular}{lcc}
\hline & At admission & At discharge \\
\hline Weight $(\mathrm{kg})$ & $47.4 \pm 9.6$ & $45.0 \pm 8.3^{*}$ \\
BMI $\left(\mathrm{kg} / \mathrm{m}^{2}\right)$ & $20.5 \pm 3.8$ & $19.4 \pm 3.5^{*}$ \\
Weight loss $(\%)$ & & $4.7 \pm 5.2$ \\
CC $(\mathrm{cm})$ & $29.0 \pm 3.0$ & $28.3 \pm 2.9^{*}$ \\
CC loss (\%) & & $2.3 \pm 3.3$ \\
Sarcopenia (n, \%) & $17(47.2)$ & $20(55.6)$ \\
Malnutrition (n, \%) & $22(61.1)$ & $24(66.7)$ \\
\hline Mean \pm SD. & \\
BMI: Body Mass Index; CC: Calf Circumference. Compari- \\
son of admission and discharge. Continuous variables: un- \\
paired t-test. Categorical variables: $\chi^{2}$ test. *p $<0.05$.
\end{tabular}

Table 3. Spearman rank correlations for Barthel Index at discharge

\begin{tabular}{lc}
\hline Variables & $\mathrm{r}$ \\
\hline Age & $-0.384^{*}$ \\
Gender & -0.047 \\
BMI & $0.377^{*}$ \\
Presence of sarcopenia at admission & $-0.460^{* *}$ \\
MNA-SF at admission & $0.650^{* *}$ \\
TP & -0.013 \\
CRP & -0.072 \\
Energy intake rates & 0.232 \\
Pain & 0.148 \\
Grade of SQ method & -0.005 \\
Number of vertebral compression fractures & -0.273 \\
The days required to begin gait training & -0.134 \\
Presence of sarcopenia at discharge & $-0.434^{* *}$ \\
MNA-SF at discharge & $0.595^{* *}$ \\
Weight loss & $-0.450^{* *}$ \\
CC loss & $-0.347^{*}$ \\
Stroke & -0.184 \\
Fracture (including other than VCF) & 0.041 \\
Heart disease & 0.062 \\
Respiratory disease & 0.069 \\
Dementia & -0.234 \\
Diabetes & -0.244 \\
\hline BMI Body Mass &
\end{tabular}

BMI: Body Mass Index; MNA-SF: Mini Nutritional AssessmentShort Form; TP: Total Protein; CRP: C-reactive protein; BI: Barthel Index; SQ: Semiquantitative Method; CC: Calf Circumference. $* \mathrm{p}<0.05, * * \mathrm{p}<0.01$.

Table 4. Multiple linear regression analysis for Barthel Index at discharge

\begin{tabular}{lrrrrr}
\hline \multirow{2}{*}{ Variables } & \multicolumn{2}{c}{ Unstandardized } & \multirow{2}{*}{ Standardized $\beta$} & p-value & 95\% CI \\
\cline { 2 - 3 } & \multicolumn{1}{c}{$\mathrm{B}$} & \multicolumn{1}{c}{$\mathrm{SE}$} & & & \\
\hline (Constant) & 115.301 & 26.946 & & $<0.001$ & 60.414 to 170.189 \\
MNA-SF at hospitalization & 5.486 & 0.766 & 0.669 & $<0.001$ & 3.925 to 7.047 \\
CC loss & -2.375 & 0.549 & -0.423 & $<0.001$ & -3.476 to -1.238 \\
Age & -0.807 & 0.324 & -0.246 & 0.018 & -1.468 to -0.147 \\
\hline
\end{tabular}

Adjusted $\mathrm{R}^{2}: 0.695, \mathrm{p}<0.001$

CI: Confidence Interval; MNA-SF: Mini Nutritional Assessment-Short Form; CC: Calf Circumference.

The concomitant development of osteoporosis and sarcopenia due to aging and malnutrition is thought to cause VCF by decreasing physical function and increasing fall and bone vulnerability ${ }^{20}$. Furthermore, patients with sarcopenia had lower ADL at discharge, and univariate analysis showed relationships of lower ADL at discharge with sarcopenia and malnutrition. Previous studies have suggested that elderly inpatients with malnutrition and/or sarcopenia have lower ADL, and we obtained similar results ${ }^{21,22}$. Therefore, sarcopenia and malnutrition are risk factors for low ADL in patients with VCF, and both of these factors should be assessed.

In this study, the losses of weight and $\mathrm{CC}$ found in many participants at discharge were closely related to the increases in sarcopenia from $47.2 \%$ at admission to $55.6 \%$ at discharge and in malnutrition from $61.1 \%$ at admission to $66.7 \%$ at discharge. A previous study found development of sarcopenia in $14.7 \%$ of inpatients ${ }^{23}$, and this may be related to nutritional status and the number of days of bed rest ${ }^{23}$. Patients with VCF are often forced to have bed rest due to pain, and in the current study, the mean period required for commencement of gait training was 12.0 days in all the participants. Therefore, malnutrition, bed rest, and lowered activity are all considered to be causes of CC loss.

The findings of this study showing that malnutrition, CC loss, and age affect ADL at discharge indicate the importance of nutritional management during rehabilitation to improve nutritional status and sarcopenia. This result also shows the 
importance of working with managerial dieticians to evaluate nutritional status and muscle mass from the point of hospital admission. In recent years, it has been suggested that a combination of rehabilitation and appropriate nutritional management are useful for improvement of sarcopenia and malnutrition ${ }^{11)}$. Even during bed rest, patients should be provided with active resistance training at the bedside and neuromuscular electrical stimulation therapy ${ }^{24,25)}$ to prevent muscle atrophy.

In this study, we utilized grip strength and CC to evaluate sarcopenia. In general, measurement of muscle mass can be made using bioelectrical impedance analysis (BIA) and dual-energy X-ray absorptiometry (DEXA) in evaluation of sarcopenia ${ }^{4}, 15$. However, few facilities have such devices available. For measurement of physical function, walking speed can be used; however, it is often difficult to measure the walking speed of inpatients with pain in an acute phase or during bed rest, as was the case for some patients in this study. A previous study found difficulty with evaluation of walking speed in $40.2 \%$ of elderly inpatients due to medical problems ${ }^{26}$. Previous results ${ }^{21,27)}$ and those in this study suggest that sarcopenia can be adequately evaluated using grip strength and $\mathrm{CC}$, and that this evaluation is useful for prognostic prediction of ADL and evaluation of nutritional status. It is also important to begin regular evaluation of grip strength and the circumferences of extremities using a simple method at admission.

The limitations of this study include the small number of participants at a single facility. Further studies are needed with more participants in multiple facilities, including examination of an intervention using a combination of nutritional management and rehabilitation for patients with VCF. Within these limitations, we conclude that many patients with VCF exhibit sarcopenia and malnutrition, and that these conditions may exacerbate during hospitalization. These changes may affect ADL, which suggests the importance of rehabilitation nutrition management for patients with VCF.

\section{Conflict of interest}

The authors report no conflicts of interest in this work.

\section{REFERENCES}

1) Harada A, Matsuyama Y, Nakano T, et al.: Nationwide survey of current medical practices for hospitalized elderly with spine fractures in Japan. J Orthop Sci, 2010, 15: 79-85. [Medline] [CrossRef]

2) Crans GG, Silverman SL, Genant HK, et al.: Association of severe vertebral fractures with reduced quality of life: reduction in the incidence of severe vertebral fractures by teriparatide. Arthritis Rheum, 2004, 50: 4028-4034. [Medline] [CrossRef]

3) Gerdhem P: Osteoporosis and fragility fractures: vertebral fractures. Best Pract Res Clin Rheumatol, 2013, 27: 743-755. [Medline] [CrossRef]

4) Cruz-Jentoft AJ, Baeyens JP, Bauer JM, et al. European Working Group on Sarcopenia in Older People: Sarcopenia: European consensus on definition and diagnosis: report of the European working group on sarcopenia in older people. Age Ageing, 2010, 39: 412-423. [Medline] [CrossRef]

5) Kim JY, Chae SU, Kim GD, et al.: Changes of paraspinal muscles in postmenopausal osteoporotic spinal compression fractures: magnetic resonance imaging study. J Bone Metab, 2013, 20: 75-81. [Medline] [CrossRef]

6) Macdonald JH, Evans SF, Davie MW, et al.: Muscle mass deficits are associated with bone mineral density in men with idiopathic vertebral fracture. Osteoporos Int, 2007, 18: 1371-1378. [Medline] [CrossRef]

7) Iolascon G, Giamattei MT, Moretti A, et al.: Sarcopenia in women with vertebral fragility fractures. Aging Clin Exp Res, 2013, 25: S129-S131. [Medline] [CrossRef]

8) Hong W, Cheng Q, Zhu X, et al.: Prevalence of sarcopenia and its relationship with sites of fragility fractures in elderly Chinese men and women. PLoS One, 2015, 10: e0138102. [Medline] [CrossRef]

9) Hida T, Shimokata H, Sakai Y, et al.: Sarcopenia and sarcopenic leg as potential risk factors for acute osteoporotic vertebral fracture among older women. Eur Spine J, 2016, 25: 3424-3431. [Medline] [CrossRef]

10) Alexandru D, So W: Evaluation and management of vertebral compression fractures. Perm J, 2012, 16: 46-51. [Medline] [CrossRef]

11) Wakabayashi H, Sashika H: Malnutrition is associated with poor rehabilitation outcome in elderly inpatients with hospital-associated deconditioning a prospective cohort study. J Rehabil Med, 2014, 46: 277-282. [Medline] [CrossRef]

12) Marshall S, Bauer J, Isenring E: The consequences of malnutrition following discharge from rehabilitation to the community: a systematic review of current evidence in older adults. J Hum Nutr Diet, 2014, 27: 133-141. [Medline] [CrossRef]

13) Otsuka K, Tazawa M, Ibe $Y$, et al.: The association between nutrition and the functional outcomes of elderly women with acute vertebral compression fractures. Kitakanto M J, 2018, 68: 1-5. [CrossRef]

14) Nakano T, Kuwabara A, Mizuta H, et al.: Contribution of hypoalbuminemia and decreased renal function to the increased mortality after newly diagnosed vertebral fracture in Japanese subjects. Asia Pac J Clin Nutr, 2016, 25: 472-477. [Medline]

15) Chen LK, Liu LK, Woo J, et al.: Sarcopenia in Asia: consensus report of the asian working group for sarcopenia. J Am Med Dir Assoc, 2014, 15: 95-101. [Medline] [CrossRef]

16) Maeda K, Koga T, Nasu T, et al.: Predictive accuracy of calf circumference measurements to detect decreased skeletal muscle mass and European Society for Clinical Nutrition and Metabolism-defined malnutrition in hospitalized older patients. Ann Nutr Metab, 2017, 71: 10-15. [Medline] [CrossRef]

17) Rubenstein LZ, Harker JO, Salvà A, et al.: Screening for undernutrition in geriatric practice: developing the short-form mini-nutritional assessment (MNASF). J Gerontol A Biol Sci Med Sci, 2001, 56: M366-M372. [Medline] [CrossRef]

18) Holden MK, Gill KM, Magliozzi MR, et al.: Clinical gait assessment in the neurologically impaired. Reliability and meaningfulness. Phys Ther, 1984, 64: 35-40. [Medline] [CrossRef]

19) Genant HK, Wu CY, van Kuijk C, et al.: Vertebral fracture assessment using a semiquantitative technique. J Bone Miner Res, 1993, 8: 1137-1148. [Medline] [CrossRef] 
20) Hida T, Harada A, Imagama S, et al.: Managing sarcopenia and its related-fractures to improve quality of life in geriatric populations. Aging Dis, 2013, 5: 226-237. [Medline]

21) Morandi A, Onder G, Fodri L, et al.: The association between the probability of sarcopenia and functional outcomes in older patients undergoing in-hospital rehabilitation. J Am Med Dir Assoc, 2015, 16: 951-956. [Medline] [CrossRef]

22) Oliveira MR, Fogaça KC, Leandro-Merhi VA: Nutritional status and functional capacity of hospitalized elderly. Nutr J, 2009, 8: 54. [Medline] [CrossRef]

23) Martone AM, Bianchi L, Abete P, et al.: The incidence of sarcopenia among hospitalized older patients: results from the Glisten study. J Cachexia Sarcopenia Muscle, 2017, 8: 907-914. [Medline] [CrossRef]

24) Wageck B, Nunes GS, Silva FL, et al.: Application and effects of neuromuscular electrical stimulation in critically ill patients: systematic review. Med Intensiva, 2014, 38: 444-454. [Medline] [CrossRef]

25) Sillen MJ, Franssen FM, Gosker HR, et al.: Metabolic and structural changes in lower-limb skeletal muscle following neuromuscular electrical stimulation: a systematic review. PLoS One, 2013, 8: e69391 [CrossRef]. [Medline]

26) Bianchi L, Abete P, Bellelli G, et al. GLISTEN Group Investigators: Prevalence and clinical correlates of sarcopenia, identified according to the EWGSOP definition and diagnostic algorithm, in hospitalized older people: the GLISTEN study. J Gerontol A Biol Sci Med Sci, 2017, 72: 1575-1581. [Medline] [CrossRef]

27) Ishii S, Tanaka T, Shibasaki K, et al.: Development of a simple screening test for sarcopenia in older adults. Geriatr Gerontol Int, 2014, 14: 93-101. [Medline] [CrossRef] 